\title{
THE RADIANCE OBSTRUCTION \\ AND PARALLEL FORMS ON AFFINE MANIFOLDS
}

BY

WILLIAM GOLDMAN AND MORRIS W. HIRSCH

\begin{abstract}
A manifold $M$ is affine if it is endowed with a distinguished atlas whose coordinate changes are locally affine. When they are locally linear $M$ is called radiant. The obstruction to radiance is a one-dimensional class $c_{M}$ with coefficients in the flat tangent bundle of $M$. Exterior powers of $c_{M}$ give information on the existence of parallel forms on $M$, especially parallel volume forms. As applications, various kinds of restrictions are found on the holonomy and topology of compact affine manifolds.
\end{abstract}

Introduction. An affine manifold $M$ is a manifold with a distinguished maximal atlas of charts, all of whose coordinate changes are locally affine. On such a manifold there is an intrinsic notion of a parallel tensor: one whose components in any affine chart are constants. More generally there is the notion of a polynomial tensor field of given degree.

In 1962, L. Markus conjectured in [Mk] that a compact orientable affine $\boldsymbol{n}$-dimensional manifold has parallel volume form if and only if it is complete (meaning that its universal covering is affinely isomorphic to Euclidean $n$-space $\mathbf{R}^{n}$ ). The problem of constructing a parallel volume form determines an $n$-dimensional twisted real cohomology class originally studied by J. Smillie [Sm2]. In this paper we express this class as the $n$th exterior power of a twisted one-dimensional real class which we call the radiance obstruction $c_{M}$. By computing $c_{M}$ in various cohomology theories-Čech, singular, de Rham, and others-we are able to exploit $c_{M}$ in several ways to yield more information on the structure of affine manifolds. Some of these results will appear in a subsequent paper [GH3].

The basic tool used is a formula, proved in \$2.6, which expresses the cohomology class of a parallel exterior $k$-form in terms of the $k$ th exterior power of the radiance obstruction, $\Lambda^{k} c_{M}$. In $\$ 2.7$ the special case of a parallel volume form on a compact $n$-dimensional manifold $M$ is examined: the existence of such a form implies that $\Lambda^{n} c_{M} \neq 0$. In $\S 2.8$ we show that the affine holonomy group $\Gamma$ of such an $M$ cannot preserve a proper affine subspace. (In [GH3] this result will be improved by showing that $\Gamma$ preserves no proper semialgebraic subset of $\mathbf{R}^{n}$.)

In $\$ 2.10$ we show that there tend to be plenty of parallel forms on a compact affine manifold with nilpotent affine holonomy.

Received by the editors May 26, 1983.

1980 Mathematics Subject Classification. Primary 57R99, 53C05; Secondary 53C10, 55R25.

c1984 American Mathematical Society $0002-9947 / 84 \$ 1.00+\$ .25$ per page 
In $\$ 2.11$ we extend slightly a theorem due originally to J. Smillie: If an affine manifold $M$ has a parallel $k$-form $\omega$ representing a nonzero cohomology class, then the affine holonomy cannot factor through a group having real cohomological dimension less than $k$. In particular if $M$ has a parallel volume form, then the real cohomological dimension (rcd) of its fundamental group is at least equal to its dimension.

$\$ 2.12$ develops another theme of Smillie's: If $M$ admits a $k$-form $\omega$ as above, then any open cover $\mathscr{U}$ of $M$ by radiant submanifolds has nerve of dimension $\geqslant k$. (See below for the definition of radiant.)

In $\$ 2.13$ we use earlier results to rule out the existence of any affine structure with parallel volume on a compact manifold of the form $B \times N$, where $N$ has finite fundamental group. If also the first Betti number of $B$ is zero, then $B \times N$ has no affine structure whatsoever.

In $\$ 2.14$ we show that the affine holonomy of a compact manifold cannot factor through certain kinds of groups studied by Margulis. For example $\operatorname{SL}(n, Z)$, for $n \geqslant 3$, is not the fundamental group of any compact affine manifold.

The earlier sections contain a systematic development of certain topological notions related to affine manifolds. It is useful to begin with affine representations of groups. If $\alpha: G \rightarrow \operatorname{Aff}(\mathbf{E})$ is such a representation (where $\mathbf{E}=\mathbf{R}^{n}$ ), we call $\alpha$ radiant if $\alpha(G)$ fixes a point of $\mathbf{E}$. The obstruction to $\alpha$ being radiant is a class $c_{\alpha} \in H^{1}\left(G ; \mathbf{E}_{\lambda}\right)$, where $\lambda: G \rightarrow \operatorname{GL}(\mathbf{E})$ is the linear part of $\alpha$. We call $c_{\alpha}$ the radiance obstruction of $\alpha$.

If $\pi$ is the fundamental group of a differentiable manifold $M$, then associated to an affine representation of $\pi$ is a flat affine bundle $\xi$ over $M$, and conversely. The radiance obstruction of $\alpha$ maps into a twisted 1-dimensional class on $M$, called $c(\xi)$, the radiance obstruction of $\xi$. The vanishing of $c(\xi)$ is equivalent to the bundle $\xi$ having a global flat section; or equivalently, to $\xi$ being isomorphic (qua flat affine bundle) to a flat vector bundle. When $c(\xi)=0$ we call $\xi$ a radiant bundle.

These radiance obstructions for bundles and representations are the subjects of $\S 1$.

In $\$ 2$ we commence the study of affine manifolds. The tangent vector bundle $T M$ of an affine manifold $M$ has a natural flat affine structure $T^{\text {aff }} M$. The radiance obstruction $c_{M}$ of $M$ is defined to be $c\left(T^{\text {aff }} M\right)$. Now $T M$ also has a natural flat vector bundle structure. The latter is isomorphic qua flat bundle to $T^{\text {aff }} M$ precisely when $c_{M}=0$.

When $c_{M}=0$ we call $M$ a radiant manifold. Geometrically this is equivalent to the existence of an atlas of affine charts whose coordinate changes are all linear.

These and other ideas are developed in \$\$2.1-2.5.

The radiance obstruction $c_{M}$ was studied in the context of cohomology of groups in Hirsch [H] and in Fried, Goldman and Hirsch [FGH2]; compare also [FGH1].

In [GH3] the radiance obstruction will be examined in Lie algebra cohomology and in algebraic group cohomology, with further application to affine structures.

\section{Flat affine bundles and cohomology.}

1.1. The radiance obstruction of an affine representation. A map $f: \mathbf{E} \rightarrow \mathbf{F}$ between real finite-dimensional vector spaces is affine if there exist a linear map $A: \mathbf{E} \rightarrow \mathbf{F}$ 
and $b \in \mathbf{F}$ such that $f(x)=A x+b$. We call $A$ the linear part and $b$ the translational part of $f$.

The bijective affine maps of $\mathbf{E}$ to itself form the group $\operatorname{Aff}(\mathbf{E})$ of affine automorphisms of $\mathbf{E}$. It is the semidirect product of the normal subgroup of translations (canonically identified with $\mathbf{E}$ ) and the subgroup GL(E) of linear automorphisms. Notice that $\mathbf{E}$ is the kernel of the homomorphism Lin: Aff(E) $\rightarrow$ GL(E) which assigns to each affine automorphism its linear part.

The map Trans: $\operatorname{Aff}(\mathbf{E}) \rightarrow \mathbf{E}$, defined by taking translational parts, is not a homomorphism (unless $\operatorname{dim} \mathbf{E}=0$ ) but a crossed homomorphism, or cocycle, with values in the identity representation of GL(E).

More generally let $\alpha: G \rightarrow \operatorname{Aff}(E)$ be an affine representation of $G$ (i.e. $\alpha$ is a homomorphism) with linear part

$$
\lambda=\operatorname{Lin} \circ \alpha: G \rightarrow \operatorname{GL}(\mathbf{E})
$$

and translational part

$$
u=\operatorname{Trans} \circ \alpha: G \rightarrow \mathbf{E} .
$$

Then $u$ is a 1-cocycle with values in the (linear) representation $\lambda$ (or in the $G$-module $\left.\mathbf{E}_{\lambda}\right)$. It therefore belongs to a cohomology class $c_{\alpha}=[u] \in H^{1}\left(G ; \mathbf{E}_{\lambda}\right)$. We call $c_{\alpha}$ the radiance obstruction of the affine representation $\alpha$. (Below we define radiance obstructions for affine bundles and affine manifolds.)

Proposition. Two affine representations having the same linear part are conjugate by a translation if and only if they have the same radiance obstruction.

Proof. Let $\lambda: G \rightarrow G L(E)$ be the linear part of affine representations $\alpha, \beta$ : $G \rightarrow \mathrm{GL}(\mathbf{E})$ whose translational parts are $u, v: G \rightarrow \mathbf{E}$. Then $c_{\alpha}=c_{\beta}$ precisely when $u-v$ is a principal crossed homomorphism, i.e. when there exists $a \in \mathbf{E}$ such that $u(g)-v(g)=a-\lambda(g) a$ for all $g \in G$. This is equivalent to $\tau_{a}^{-1} \alpha(g) \tau_{a}=\beta(g)$, where $\tau_{a}$ denotes translation by $a$. Q.E.D.

COROLlary. An affine representation has a stationary point if and only if the radiance obstruction vanishes.

1.2. Holonomy of flat bundles. Let $\xi=(p, E, B, G, X, \Phi)$ be a fibre bundle with total space $E$, base $B$, projection $p: E \rightarrow B$, topological structure group $G$ acting effectively on the standard fibre $X$, and structure atlas $\Phi$.

To say that $\Phi$ is an atlas means that $\Phi$ is a set of homeomorphisms $\Phi_{i}$ : $p^{-1} U_{i} \rightarrow U_{i} \times X$ (indexed for convenience), where $\left\{U_{i}\right\}$ is an open cover of $B$. For $x \in U_{i}$ define $\Phi_{i, x}: p^{-1}(x) \rightarrow X$ by $\Phi_{i}(y)=\left(x, \Phi_{i, x} y\right)$. There are continuous transition maps $g_{i j}: U_{i} \cap U_{j} \rightarrow G$ such that for $x \in U_{i} \cap U_{j}$, the homeomorphism $\Phi_{i, x} \Phi_{j, x}^{-1}$ : $X \rightarrow X$ coincides with the action of $g_{i j}(x)$. When $G$ acts effectively on $X$ we have, for $x \in U_{i} \cap U_{j} \cap U_{k}$,

$$
g_{i j}(x) g_{j k}(x) g_{k i}(x)=1 \in G
$$

(the identity element) and also $g_{i i}(x)=1$.

A structure atlas is a maximal atlas. 
We call $\xi$ a flat bundle if $G$ is a discrete group. In this case the transition maps are locally constant. There is then a subatlas whose transition maps $g_{i j}$ are constant, i.e., $g_{i j} \in G$. If $G$ acts effectively we obtain a family $\left\{g_{i j}\right\}$ of elements of $G$ satisfying $g_{i j} g_{i k} g_{k i}=1=g_{i i}$. We call such a family a cocycle with values in $G$.

Suppose now that $G$ is not necessarily discrete. Let $G^{\delta}$ be the group $G$ with the discrete topology. If $\xi$ is a bundle with structure group $G$, a flat structure on $\xi$ is a subatlas $\Phi_{0}$ of the structure atlas $\Phi$ such that $\Phi_{0}$ is the structure atlas for a $G^{\delta}$ bundle. In other words, a flat structure on $\xi$ is determined by an atlas having locally constant transition maps. The same bundle can have many inequivalent flat structures.

Let $\xi$ be a flat bundle as above. A section $s: U \rightarrow E$ of $\xi$ over an open set $U \subset B$ is called flat (or parallel) if for every local trivialization $\left(\phi_{i}, U_{i}\right)$ the composition

$$
U \cap U_{i} \rightarrow p^{-1} U_{i} \stackrel{\phi_{i}}{\rightarrow} U_{i} \times X \rightarrow X
$$

is locally constant.

If $U \subset U_{i}$ is a connected open set and $y \in p^{-1} U$, then there is a unique flat section over $U_{i}$ through $y$, namely the composition

$$
U \rightarrow U_{i} \stackrel{(\text { id, } y)}{\rightarrow} U_{i} \times Y \stackrel{\phi_{i}^{-1}}{\rightarrow} p^{-1} U_{i}
$$

It is readily proved that $\xi$ has a global flat section if and only if its structure group can be reduced to a subgroup fixing a point of $X$.

Suppose now that the flat bundle $\xi$ is a smooth bundle: $E, B$ and $X$ are manifolds, $G$ acts on $X$ by diffeomorphisms and the maps $p, \phi_{i}$ are smooth (we need only consider the $C^{\infty}$ case). Then there is a unique foliation $\mathscr{F}=\mathscr{F}(\xi)$ of $E$ obtained as follows: If $\left(\phi_{i}, U_{i}\right) \in \Phi$ and $U_{i}$ is connected, then $\mathscr{F} \mid p^{-1} U_{i}$ has as leaves the images of the flat sections over $U_{i}$. These foliations induce the same foliation on $p^{-1} U_{i} \cap p^{-1} U_{j}$; thus $\mathscr{F}$ is well defined.

It is easy to see that each leaf $L$ of $\mathscr{F}$, in its manifold topology, is a covering space of $B$ via $p \mid L: L \rightarrow B$; and the inclusion $L \rightarrow E$ is an immersion transverse to the fibres of $\xi$.

Let $\lambda:[0,1] \rightarrow B$ be a path from $b_{0}$ to $b_{1}$. Given a point $y$ in the fibre $E_{b_{0}}$ over $b_{0}$, there is a unique lift $\lambda_{y}:[0,1] \rightarrow E$ of $\lambda$ into the leaf through $y$ such that $\lambda_{y}(0)=y$ and $p \circ \lambda_{y}=\lambda$. Define $h_{\lambda}(y)=\lambda_{y}(1)$. In this way a map $h_{\lambda}: E_{b_{0}} \rightarrow E_{b_{1}}$ is obtained. The covering homotopy property for covering spaces ensures that $h_{\lambda}$ depends only on the homotopy class (rel endpoints) $[\lambda]$ of $\lambda$. In local trivializations $h_{\lambda}$ appears as a diffeomorphism of $X$ corresponding to some element of $G$.

It is easy to see that when $b_{0}=b_{1}=b$ we obtain a homomorphism

$$
h_{b}: \pi_{1}(B, b) \rightarrow \operatorname{Diff}\left(E_{b}\right)
$$

into the group of diffeomorphisms of $E_{b}$, defined by $h_{b}([\lambda])(y)=h_{\lambda}(y)$.

For $\left(\Phi_{i}, U_{i}\right) \in \Phi$ and $b \in U_{i}$ we define a homomorphism

$$
h_{i, b}: \pi_{1}(B, b) \rightarrow \operatorname{Diff}(Y), \quad[\lambda] \mapsto \phi_{i, b} \circ h_{b}(\lambda) \circ \phi_{i, b}^{-1} .
$$


Then the image of $h_{i, b}$ lies in the image of $G$. When $G$ acts effectively we can uniquely lift $h_{i, b}$ into $G$, obtaining a homomorphism $\pi_{1}(B, b) \rightarrow G$.

Now let $g: \tilde{B} \rightarrow B$ be a universal cover and let $\pi \subset$ Diff $\tilde{B}$ be its group of deck transformations. Identify $\pi$ with $\pi_{1}(B, b)$ in the usual way (recall this depends on choosing a base point in $\left.g^{-1}(b)\right)$. There results a homomorphism $h: \pi \rightarrow G$ which depends on the choice of $b$ and the choice of $\left(\phi_{i}, U_{i}\right)$. If $h^{\prime}: \pi \rightarrow G$ is the homomorphism corresponding to different choices, then there exists a unique $g \in G$ such that $h^{\prime}$ is the composition of $h$ with conjugation by $g$. By abuse of language we call $h$ the holonomy of $\xi$.

Conversely, every homomorphism $h: \pi \rightarrow G$ determines a canonical flat bundle $\eta=\eta(h)$ with holonomy $h$ : the total space $E(\eta)$ of $\eta$ is the quotient of $\tilde{B} \times X$ by the diagonal action of $\pi$. The leaves of the foliation $\mathscr{F}(\eta)$ are the images in $E(\eta)$ of the sets $\tilde{B} \times\{x\}$. If $h$ is the holonomy of $\xi$, then $\xi$ and $\eta(h)$ are canonically isomorphic bundles.

1.3. Affine bundles, vector bundles, derived bundles. Let $\mathbf{E}$ denote the vector space $\mathbf{R}^{n}$. A bundle with standard fibre $\mathbf{E}$ is an affine bundle if the structure group is $\operatorname{Aff}(\mathbf{E})$; it is a vector bundle if the group is $\operatorname{GL}(\mathbf{E})$.

Fix an affine bundle $\xi=(p, E, B)$. A translation of a fibre $E_{b}, b \in B$, is a map $E_{b} \rightarrow E_{b}$ which corresponds to a translation of $\mathbf{E}$ when $E_{b}$ and $\mathbf{E}$ are identified by a local trivialization. The set of translations of $E_{b}$ is a group under composition, isomorphic to $\mathbf{E}$ (but not canonically) via local trivialization. Thus the translations of $E_{b}$ form a vector space, denoted by $E_{b}^{L}$.

Let $E^{L}=\bigcup_{b \in B} E_{b}^{L}$. Define $p^{L}: E^{L} \rightarrow B$ by sending $E_{b}^{L}$ to $b$. In a natural way this map is the projection of a vector bundle, called the derived bundle $\xi^{L}$ of $\xi$.

Suppose $\xi$ is a flat affine bundle with a cocycle $\left\{g_{i j}\right\}$ and a holonomy homomorphism $h: \pi \rightarrow \operatorname{Aff}(\mathbf{E})$. Then $\xi^{L}$ is a flat vector bundle, with a cocycle $\left\{\operatorname{Lin}\left(g_{i j}\right)\right\}$ and a holonomy homomorphism $h^{\prime}=\operatorname{Lin} \circ h: \pi \rightarrow \mathrm{GL}(\mathrm{E})$. We also call $h^{\prime}$ the linear holonomy of $\boldsymbol{\xi}$.

1.4. Radiant bundles. A subtle question of structure arises here. Every vector bundle can be considered an affine bundle by means of the natural inclusion $i$ : $\operatorname{GL}(\mathbf{E}) \rightarrow \operatorname{Aff}(\mathbf{E})$. Since the composition $i \circ \operatorname{Lin}: \operatorname{Aff}(\mathbf{E}) \rightarrow \operatorname{Aff}(\mathbf{E})$ is homotopic to the identity through homomorphisms, it follows that an affine bundle $\xi$ is isomorphic, as an affine bundle, to its derived bundle $\xi^{L}$. An explicit affine isomorphism $F: \xi \rightarrow \xi^{L}$ is easily defined. Let $s: B \rightarrow \xi$ be any section of $\xi$. For $b \in B$ and $y \in \xi_{b}$ let $F(y)$ be the unique translation of $\xi_{b}$ taking $s(b)$ to $y$. Then $F: \xi \rightarrow \xi^{L}$ is an isomorphism of affine bundles.

When $\xi$ is a flat affine bundle, however, $\xi^{L}$ is a flat vector bundle which is not generally isomorphic to $\xi$ as a flat bundle. In later sections we will measure the difference between a flat affine bundle and its derived flat vector bundle by a cohomology class. The following result describes the case where they are isomorphic:

Proposition. Let $\xi$ be a flat affine bundle. Then the following conditions are equivalent:

(a) $\xi$ has a flat section;

(b) $\xi$ is isomorphic to $\xi^{L}$ as a flat affine bundle; 
(c) $\xi$ is isomorphic to some flat vector bundle considered as an affine bundle;

(d) the affine holonomy representation $h: \pi \rightarrow \operatorname{Aff}(E)$ of $\xi$ has a stationary point in E.

Proof. The affine isomorphism $\xi \rightarrow \xi^{L}$ constructed above from a section of $\xi$ is an isomorphism of flat bundles if the section is flat. Thus (a) $\Rightarrow(b)$, and (b) $\Rightarrow$ (c) is obvious. Since every flat vector bundle has a flat section, namely its zero section, (c) $\Rightarrow$ (a). Finally (a) $\Leftrightarrow(d)$, since flat sections correspond to stationary points of the affine holonomy representation $\pi \rightarrow \operatorname{Aff}(\mathbf{E})$. Q.E.D.

$A$ radiant bundle is defined to be a flat affine bundle $\xi$ enjoying properties (a)-(d) of the proposition above.

It is easy toconstruct nonradiant flat affine bundles. An example is the bundle over the circle $S^{1}$ corresponding to any homomorphism $\pi_{1}\left(S^{1}\right)=Z \rightarrow \operatorname{Aff}(E)$ generated by an affine automorphism without a stationary point.

1.5. Embedding affine bundles in vector bundles. It is sometimes useful to embed an affine $\xi$ bundle in a vector bundle $\xi^{J}$ having one more fibre dimension. Let $\Phi=\left\{\phi_{i}, U_{i}\right\}$ be the affine bundle structure on $\xi=(p, E, B, \operatorname{Aff}(\mathbf{E}), \mathbf{E}, \Phi)$. Define a bundle structure on the composite map

$$
g: E \times \mathbf{R} \rightarrow E \stackrel{p}{\rightarrow} B
$$

as follows. Given $i$ and $x \in U_{i}$ define

$$
\psi_{i, x}: E_{x} \times \mathbf{R} \rightarrow E \times \mathbf{R}, \quad(y, t) \mapsto\left(t \phi_{i, x}(y), t\right) .
$$

The resulting atlas $\left\{\psi_{i}, U_{i}\right\}$ defines a vector bundle

$$
\xi^{J}=\{q, E \times \mathbf{R}, \mathrm{GL}(\mathbf{E} \times \mathbf{R}), \mathbf{E} \times \mathbf{R}, \boldsymbol{\Phi}\} .
$$

Evidently $\boldsymbol{\xi}$ is isomorphic as an affine bundle to the subbundle whose total space is $E \times\{1\}$; and the derived bundle $\xi^{L}$ is isomorphic as a vector bundle to the subbundle $E \times\{0\}$.

When $\xi$ is flat so is $\xi^{J}$, and the holonomy of $\xi^{J}$ is the composition of the holonomy of $\xi$ with the homomorphism

$$
J: \operatorname{Aff}(\mathbf{E}) \rightarrow \operatorname{GL}(\mathbf{E} \times \mathbf{R}), \quad J(f):(x, t) \rightarrow(A x+t b, t),
$$

where $g(x)=A x+b$. Similarly $J$ converts a cocycle for $\xi$ to a cocycle for $\xi^{J}$.

1.6. Affine spaces. For simplicity we took the standard fibre of an affine bundle to be a vector space. In a more puristic approach it would be an affine space, that is, a set together with a free transitive action on it by a vector group. While this approach seems unnecessarily elaborate, the notions of affine spaces and affine maps between them are occasionally useful.

1.7. The radiance obstruction of a flat affine bundle. Let $\xi$ be a flat affine bundle with holonomy $h: \pi \rightarrow \operatorname{Aff}(\mathbf{E})$. We define the radiance obstruction $c(\xi)$ to be $c_{h} \in H^{1}\left(\pi ; E_{\lambda}\right)$, where $\lambda=\operatorname{Lin} \circ h: \pi \rightarrow \operatorname{GL}(\mathbf{E})$ is the linear holonomy of $\xi$, and $c_{h}$ is the radiance obstruction of $h$. As an immediate consequence of Proposition 1.5 and Corollary 1.1 we obtain

Proposition. $\xi$ has a flat section if and only if $c(\xi)=0$. 
Technically speaking, $c(\xi)$ is not well defined since $h$ and $\lambda$ are not uniquely determined; it would be more exact to write $c(\xi, h)$. But the vanishing of $c(\xi)$ is independent of the choice of $h$. One of our main goals is to express $c(\xi)$ in other cohomology theories, some of which are more intrinsic.

1.8. The singular radiance obstruction. Let $\xi$ be a flat affine bundle over a connected manifold $M$. Let $\xi^{L}$ be its derived flat vector bundle. There is a sheaf $\mathscr{S}\left(\xi^{L}\right)=\mathscr{S}$ of (germs of) flat sections of $\xi^{L}$. The total space of $\mathscr{S}$, considered as a set, can be canonically identified with the total space of $\xi^{L}$. Clearly $\mathscr{S}$ is a locally constant sheaf of vector spaces isomorphic to $\mathbf{E}$.

We consider $\mathscr{S}$ as a system of local coefficients on $M$. If we fix a base point $b \in M$ and identify the stalk of $\mathscr{S}_{b}$ over $b$ with $\mathbf{E}$, then $\mathscr{S}$ is determined by a homomorphism $\pi_{1}(M ; b) \rightarrow G L(E)$. It is easy to verify that this homomorphism corresponds to the linear holonomy $\lambda$ of $\xi$ when $\pi_{1}(M, b)$ is identified with the group $\pi$ of deck transformations of $\tilde{M}$.

Such an identification also determines a homomorphism from the cohomology of $\pi$ with coefficients in $\mathrm{E}_{\lambda}$ to the singular cohomology $H_{\text {ring }}^{*}(M ; \mathscr{S})$ of $M$ with coefficients in the local coefficient system. This homomorphism is an isomorphism of $H^{1}$. The radiance obstruction $c(\xi)$ thus corresponds to an element

$$
c_{\text {sing }}(\xi) \in H^{1}(M ; \mathscr{S}) \text {. }
$$

It is easy to verify that $c_{\text {sing }}(\xi)$ is intrinsically defined by $\xi$; the various choices made in its definition cancel out.

1.9. The Čech radiance obstruction. Let $H_{\mathrm{Cech}}^{*}(M ; \mathscr{S})$ denote the Čech cohomology of $M$ with coefficients in the sheaf $\mathscr{S}$.

There is a canonical isomorphism

$$
H_{\text {sing }}^{*}(M ; \mathscr{S}) \approx H_{\text {Cech }}^{*}(M ; \mathscr{S}) .
$$

(see Bredon [Br]). We describe a Čech 1-cocycle belonging to the image of $c_{\text {sing }}(\xi)$.

A Čech 1-chain with values in $\mathscr{S}$ is determined by an open cover $\mathscr{U}$ of $M$ together with a function assigning to each pair $U_{i}, U_{j}$ in $\mathscr{U}$ a section of $\mathscr{S}$ over $U_{i} \cap U_{j}$. Let $\mathscr{U}=\left\{U_{i}\right\}_{i \in \Lambda}$ be a radiant cover: an open cover by sets $U_{i}$ such that $\xi \mid U_{i}$ is radiant. For each $i$ let $a_{i}: U_{i} \rightarrow \xi$ be a flat section. If $x \in U_{i} \cap U_{j}$ let $t_{i j}(x) \in \xi_{x}^{L}$ be the translation of $\xi_{x}$ which takes $s_{i}(x)$ to $s_{j}(x)$. Then $t_{i j}$ is a flat section of $\xi_{L} \mid U_{i} \cap U_{j}$. This means $t_{i j}$ is a section of $\mathscr{S}_{\text {over }} U_{i} \cap U_{j}$.

It is not hard to verify that the collection $\left\{t_{i j}\right\}_{i \in \Lambda}$ is a cocycle and the resulting cohomology class $c_{\text {Cech }}(\xi)$ corresponds to $c_{\text {sing }}(\xi)$.

1.10. The standard connection on a flat vector bundle. A (linear) connection on $E$ is a supplementary bundle $\eta \subset T E$ to $p^{*} \xi$; thus $T E=\eta \oplus p^{*} \xi$. Given a connection, for any (smooth) section $s: M \rightarrow E$ we define a morphism (= bundle map) $\nabla s$ : $T M \rightarrow E$ (over the identity map $1_{M}$ ), by forming the composition

$$
\nabla s: T M \stackrel{T f}{\rightarrow} T E \stackrel{j}{\rightarrow} p^{*} E \stackrel{q}{\rightarrow} E,
$$

where $j$ is the retraction with kernel $\eta$ and $g$ is the canonical map. In this way we obtain a linear map $\nabla$ from sections of $E$ to morphisms $T M \rightarrow E$, or equivalently, to sections of $T^{*} M \otimes E$. One often identifies the connection with the map $\nabla$. 
The connection $\eta$ is called flat if $E$ has a foliation $\mathscr{G}$ whose leaves are transverse to the fibres of $E$, such that $\eta$ is the bundle $T \mathscr{G}$ of tangent planes to leaves. Every such foliation of $E$ determines a flat connection on $E$.

Now suppose that $E$ is a flat vector bundle. The canonical foliation $\mathscr{F}$ of $E$, corresponding to the flat structure, determines a flat connection on $E$. We call this the standard connection on the flat vector bundle $E$.

It is clear from the definition that if the section $s$ is tangent at $x \in M$ to a leaf of $\mathscr{F}$ then $\nabla s_{x}: T_{x} M \rightarrow E_{x}$ is zero. Thus $\nabla s$ measures the deviation of $s$ from being flat. In particular $\nabla s$ vanishes if and only if $s$ is a flat section.

A connection on the trivial vector bundle $\varepsilon=(M \times \mathbf{E}, M, \mathbf{E})$ is the same thing as a morphism $\delta: T M \rightarrow \mathbf{E}$, i.e. a map linear on fibres. To see this, recall that if $(x, y) \in M \times \mathbf{E}$, then $T_{(x, y)}(M \times \mathbf{E})$ is identified with $T_{x} M \oplus \mathbf{E}$. The subspace complementary to $0 \oplus \mathbf{E}$ is the graph of $\delta_{x}$. This connection is flat precisely when $\delta$ is closed as an E-valued 1 -form on $M$, that is, when locally $\delta$ coincides with differentials of maps from open sets of $M$ into $\mathbf{E}$.

1.11. De Rham cohomology with coefficients in a flat vector bundle. Let $\mathbf{E}, \mathbf{F}$ be real, finite-dimensional vector spaces and $U \subset \mathbf{F}$ an open set. Sections of $U \times \mathbf{E}$ correspond to maps $U \rightarrow \mathbf{E}$. The standard connection assigns to such a map $f$ a morphism $T U=U \times \mathbf{F} \rightarrow U \times \mathbf{E}$, which corresponds to a map from $U$ to the vector space $L(\mathbf{E}, \mathbf{F})$ of linear maps from $\mathbf{E}$ to $\mathbf{F}$. This map $U \rightarrow L(\mathbf{E}, \mathbf{F})$ is just the differential $d f$.

For each integer $k \geqslant 0$ the exterior differential operator defines a linear map

$$
d(k, U, \mathbf{E})=d_{k}: C^{\infty}\left(U,\left(\Lambda^{k} \mathbf{F}^{*}\right) \otimes \mathbf{E}\right) \rightarrow C^{\infty}\left(U,\left(\Lambda^{k+1} \mathbf{F}^{*}\right) \otimes \mathbf{E}\right)
$$

such that $d_{k+1} \circ d_{k}=0$; here $C^{\infty}$ indicates the vector space of $C^{\infty}$ maps.

Now let $p: E \rightarrow M$ be a flat vector bundle. By identifying fibres of $E$ with $\mathbf{E}$, and coordinate neighborhoods in $M$ with open sets $V_{i}$ in $\mathbf{F}$, we can piece together the exterior differentials $d\left(k, V_{i}, \mathbf{F}\right)$ to obtain a family of linear maps

$$
\left(d_{\nabla}\right)_{k}: \Lambda^{k}(M ; E) \rightarrow \Lambda^{k+1}(M ; E)
$$

for each integer $k \geqslant 0$. Here $\Lambda^{k}(M ; E)$ is the vector space of $E$-valued exterior $k$-forms on $M$, that is, the space of sections of the vector bundle $\Lambda^{k}\left(T^{*} M\right) \otimes E$.

Notice that $\Lambda^{0}(M ; E)$ is the space of sections of $E, \Lambda^{1}(M ; E)$ is the space of morphisms $T M \rightarrow E$, and $\left(d_{\nabla}\right)_{0}$ is just the connection $\nabla$. Thus we have extended $\nabla$ to a sequence of linear maps

$$
\begin{aligned}
0 & \rightarrow \Lambda^{0}(M ; E) \stackrel{\nabla}{\rightarrow} \cdots \rightarrow \Lambda^{k}(M ; E) \stackrel{\left(d_{\nabla}\right)_{k}}{\rightarrow} \Lambda^{k+1}(M ; E) \\
& \rightarrow \cdots \rightarrow \Lambda^{n}(M ; E) \rightarrow 0 \quad(n=\operatorname{dim} M) .
\end{aligned}
$$

Working locally one sees that $\left(d_{\nabla}\right)_{k+1} \circ\left(d_{\nabla}\right)_{k}=0$, since this holds for the exterior differential. (This uses flatness of $E$.) Therefore the sequence above is a cochain complex. Its cohomology is, by definition, the de Rham cohomology of $M$ with coefficients in the flat vector bundle $E$, denoted by $H^{*}(M ; E)$.

1.12. The covariant differential for flat affine bundles. Let $p: E \rightarrow M$ be a flat affine bundle, with its derived flat vector bundle $E^{L}$. Although $E$ is not a vector bundle we can still define a covariant differential

$$
\nabla: C^{\infty}(E) \rightarrow C^{\infty}\left(T^{*} M \otimes E^{L}\right)=\Lambda^{1}\left(M ; E^{L}\right)
$$


from sections of $E$ to morphisms $T M \rightarrow E^{L}$, in a generalization of $\S 1.10$. There is a natural affine structure (see $\S 1.6)$ on $C^{\infty}(E)$, and $\nabla$ is an affine map.

We split $T E=T \mathscr{F} \oplus T \mathscr{G}$ as in the preceding section, and remark that $T \mathscr{G}$ is naturally isomorphic to $p^{*} E^{L}$. For an element of $T \mathscr{G}$ is a tangent vector to a fibre $E_{x}$ of $E$. This fibre is an affine space (see \$1.6) and a tangent vector to an affine space corresponds naturally to a translation via affine identifications with a vector space. Thus for $y \in E_{x}$ there is a natural identification $T_{y} E_{x}=\left(E_{x}\right)^{L}$. Noting that $x=$ $p(\mathscr{G})$, in this way we identify $T \mathscr{G}=p^{*} E^{L}$.

Now define $\pi_{\nabla}$ to be the composite vector bundle morphism

$$
\pi_{\nabla}: T E=T \mathscr{F} \oplus T \mathscr{G} \rightarrow T \mathscr{G}=p^{*} E^{L} \rightarrow E^{L} .
$$

For any section $s: M \rightarrow E$ define $\nabla s$ to be the composite morphism

$$
\nabla s: T M \stackrel{T_{s}}{\rightarrow} T E \stackrel{\pi}{\rightarrow} E^{L} .
$$

As before $\nabla s=0$ precisely when $s$ is flat.

1.13. Computation of $\nabla$ for a special flat affine bundle. Fix a smooth map $g$ : $M \rightarrow \mathbf{E}$. There is a flat affine bundle $E(g)$ whose projection is the natural one, $p$ : $M \times \mathbf{E} \rightarrow M$, but whose foliation $\mathscr{F}$ has as leaves the graphs of the maps $g+$ constant. It is easy to see that there is a canonical identification of the derived flat vector bundle $E(g)^{L}$ with the trivial flat vector bundle $M \times \mathbf{E}$ (whose foliation has for leaves the graphs of constant maps).

Let $s: M \rightarrow E(g)$ be a section corresponding to $f: M \rightarrow \mathbf{E}$. It turns out that $\nabla s$ : $T M \rightarrow M \times E$ is given as follows: If $x \in M$ and $y \in T_{x} M$, then

$$
(\nabla s)_{x} y=\left(x, d f_{x} y-d g_{x} y\right) \text {. }
$$

This is proved first for the case where $g$ is identically 0 . The general case follows by considering the isomorphism of flat affine bundles

$$
E(g) \rightarrow E(0)=M \times \mathbf{E}, \quad(x, y) \mapsto(x, y-g(x))
$$

and pulling back the information on $E(0)$.

In the special case where $M=U \subset \mathbf{E}$ is an open set and $g: U \rightarrow \mathbf{E}$ is $g(x)=-x$, it follows that $\nabla s: T U=U \times \mathbf{E} \rightarrow U \times \mathbf{E}$ is given by $(x, y) \rightarrow\left(x, d f_{x}(y)+y\right)$, where $s(x)=(x, f(x)) \in U \times \mathbf{E}$. In particular, when $s$ is the zero section, $\nabla s$ is the identity map of $U \times \mathbf{E}$.

1.14. The de Rham radiance obstruction. Let $\xi=(p, E, M)$ be a flat affine bundle, $E^{L}$ its derived flat vector bundle, and $\mathscr{S}$ the sheaf of flat sections of $E^{L}$. The singular radiance obstruction $c_{\text {sing }}(\xi)$ is a class in $H_{\text {sing }}^{*}(M ; \mathscr{S})($ see $\S 1.8)$. In this section we compute the image of $c_{\text {sing }}(\xi)$ in de Rham cohomology $H^{*}\left(M ; E^{L}\right)$ (see $\left.\$ 1.11\right)$ under the canonical isomorphism. We denote this image by $c_{\xi} \in H^{1}\left(M ; E^{L}\right)$; in most later computations we shall use this form of the radiance obstruction.

Recall from $\S 1.11$ that $H^{*}\left(M ; E^{L}\right)$ is the cohomology of a cochain complex

$$
\Lambda^{k}\left(M ; E^{L}\right) \stackrel{d \nabla}{\rightarrow} \Lambda^{k+1}\left(M ; E^{L}\right) .
$$

From $\$ 1.12$ let $\nabla: C^{\infty}(E) \rightarrow \Lambda^{1}\left(M ; E^{L}\right)$ be the covariant differential.

THEOREM. Let $s: M \rightarrow E$ be any section. Then $\nabla s$ is a cocycle whose cohomology class $[\nabla s]$ is $c_{\xi}$. 
Proof. Locally $\nabla s$ is the differential of a map $U \rightarrow \mathbf{E}$. Thus $\nabla s$ is locally exact, so it is closed.

The cohomology of $M$ pulls back injectively to that of its 1-skeleton. Therefore to prove $[\nabla s]=c_{\xi}$ it suffices to prove $g^{*}[\nabla s]=g^{*} c_{\xi}$ for every map $g: S^{1} \rightarrow M$ of a circle into $M$. Now $\nabla$ and $c_{\xi}$ behave naturally for induced bundles. Therefore it suffices to prove the theorem for the special case where $M=S^{1}$.

Let $h: \pi_{1}\left(S^{1}\right) \rightarrow \operatorname{Aff}(\mathbf{E})$ be the holonomy of $\xi$. We may assume $\xi$ is the canonical flat bundle with holonomy $h$ (see $\$ 1.2$ ). Thus we take the total space $E$ to be the identification space of $\mathbf{R} \times \mathbf{E}$ by the relation

$$
(x+m, y)=\left(x, A^{m} y\right) \quad(m \in \mathbf{Z})
$$

for some $A \in \mathrm{GL}(\mathbf{E})$. A section $s: S^{1} \rightarrow E$ lifts to a map $\mathbf{R} \rightarrow \mathbf{R} \times \mathbf{E}$ of the form $x \mapsto(x, f(x))$, where $f: \mathbf{R} \rightarrow \mathbf{E}$ satisfies $f(x+1)=A f(x)$. The $\xi^{L}$-valued 1 -form $\nabla s$ corresponds to an $\tilde{E}$-valued 1 -form on $\mathbf{R}$, namely $d x \otimes d f / d s$. The de Rham homomorphism on cochains carries the 1-cocycle $\nabla s$ to the singular 1-cocycle $z$ which when applied to a differentiable singular 1-simplex $\sigma:[0,1] \rightarrow M$ gives

$$
\begin{aligned}
z(\sigma) & =\int_{[0,1]} \sigma^{*}(\nabla s)=\int_{0}^{1} \frac{\partial(f \circ \sigma)}{\partial t} d t \\
& =\int_{0}^{1} \frac{\partial \tilde{f}}{\partial r} d r=\tilde{f}(\sigma(1)-\sigma(0))
\end{aligned}
$$

If $\sigma$ is the fundamental 1 -cycle $[0,1] \rightarrow \mathbf{R} / \mathbf{Z}$ induced by the restriction of the identity map, then $z(\sigma)=\tilde{f}(1)-\tilde{f}(0)=b$ is the translational part of the affine holonomy of $\xi$. By $\S 1.8, \nabla s$ represents the radiance obstruction $c_{\xi}$ in de Rham theory. Q.E.D.

1.15. Exterior powers of the radiance obstruction. Let $E \rightarrow M$ be a flat vector bundle. For any integers $k, l \geqslant 0$ there is a natural morphism $\left(\Lambda^{k} E\right) \otimes\left(\Lambda^{l} E\right) \rightarrow$ $\Lambda^{k+l} E$. These fit together to induce bilinear maps

$$
H^{k}\left(M ; \Lambda^{k} E\right) \times H^{l}\left(M ; \Lambda^{\prime} E\right) \rightarrow H^{k+l}\left(M ; \Lambda^{k+l} E\right),
$$

and similarly with more factors on the left. In particular there is a $k$-linear map

$$
H^{1}(M ; E) \times \cdots \times H^{1}(M ; E) \rightarrow H^{k}\left(M ; \Lambda^{k} E\right) .
$$

Preceding this with the diagonal map

$$
H^{1}(M ; E) \rightarrow H^{1}(M ; E) \times \cdots \times H^{1}(M ; E)
$$

gives the exterior $k$ th power map $\Lambda^{k}: H^{1}(M ; E) \rightarrow H^{k}\left(M ; \Lambda^{k} E\right)$.

Now, changing notation, let $\xi=(p, E, M)$ be a flat affine bundle and consider $\Lambda^{k} c_{\xi} \in H^{k}\left(M ; \Lambda^{k}\left(E^{L}\right)\right)$ in de Rham theory.

THEOREM. If $\xi$ has a flat affine subbundle of fibre dimension $j$, then $\Lambda^{k} c_{\xi}=0$ for all $k>j$.

Proof. Let $\eta=(p, F, M)$ be the subbundle. Viewing the inclusion $i: F^{L} \subset E^{L}$ as a coefficient homomorphism, we derive an induced homomorphism

$$
i_{\#}: H^{*}\left(M ; F^{L}\right) \rightarrow H^{*}\left(M ; E^{L}\right) \text {. }
$$


One easily verifies that $i_{\#} c_{\eta}=c_{\xi}$, for example by using $\S 1.14$. Since $\Lambda^{k} F^{L}$ has zero-dimensional fibres for $k>j$, the theorem follows. Q.E.D.

Below we give various conditions ensuring $\Lambda^{k} c_{\xi} \neq 0$.

2. The radiance obstruction of an affine manifold. Now we apply the preceding theory of flat affine bundles to affine manifolds. Recall that an affine structure on a (differentiable) manifold $M$ is defined as follows. Let $\mathbf{E}$ be a fixed vector space having the same dimension as $M$. Let $U \subset M$ be an open set and let $\psi: U \rightarrow \mathbf{E}$ be a coordinate chart for the manifold structure on $M$. Two coordinate charts $\psi_{i}: U_{i} \rightarrow \mathbf{E}$ $(i=1,2)$ are said to be (affinely) compatible if on each component of $\psi_{1}\left(U_{1}\right) \cap \psi_{2}\left(U_{2}\right)$ the map $\psi_{2}^{-1} \circ \psi_{1}$ extends to an affine automorphism of $E$. In other words, the coordinate change $g_{12}=\psi_{1} \psi_{2}^{-1}: \psi_{2}\left(U_{1} \cap U_{2}\right) \rightarrow \psi_{1}\left(U_{1} \cap U_{2}\right)$ is locally affine. An affine structure is defined to be a maximal atlas of compatible affine coordinate charts. An affine manifold is a manifold with an affine structure.

Throughout the rest of this work, $M$ denotes a connected affine manifold modeled on the vector space $\mathbf{E}=\mathbf{R}^{n}, \boldsymbol{n}>0$.

2.1. Flat structures on the tangent bundle. Let $M$ be an affine manifold. As a differential manifold, $M$ has a tangent vector bundle $T M$. To the affine structure we shall associate two other bundle structures on $T M$ : a flat affine bundle $T^{\text {aff }} M$, and a flat vector bundle canonically identified with the derived bundle $\left(T^{\text {aff }} M\right)^{L}$. Thus $T M$ has three bundle structures; it is important to keep them conceptually distinct.

If $f: M \rightarrow N$ is an affine map between affine manifolds then we will see that the tangent map $T f: T M \rightarrow T N$ is a morphism for each of the three bundle structures. It also turns out that the natural (identity) map $T M \rightarrow T^{\text {aff }} M$ is an isomorphism of affine bundles, while the natural map $\left(T^{\text {aff }} M\right)^{L} \rightarrow T M$ is an isomorphism of vector bundles. The natural map $T^{\text {aff }} M \rightarrow\left(T^{\text {aff }} M\right)^{L}$ is an isomorphism of affine bundles; it is not an isomorphism of flat bundles unless $M$ is radiant.

We now define a flat affine structure for $T M$. Let $\left\{\phi_{i}, U_{i}\right\}$ be an affine atlas for $M$ modeled on $\mathbf{E}=\mathbf{R}^{n}$. For each $i$ and each $x \in U_{i}$ define an affine isomorphism

$$
\theta_{i, x}: T_{x} M \rightarrow \mathbf{E}, \quad v \mapsto \phi_{i}(x)+d \phi_{i}(x) v .
$$

Define the natural affine trivializations

$$
\boldsymbol{\theta}_{i}: T U_{i} \rightarrow U \times \mathbf{E}, \quad v \mapsto\left(x, \theta_{i, x}(v)\right) \quad \text { if } v \in T_{x} M .
$$

One easily sees that $\left\{\theta_{i}, U_{i}\right\}$ is an atlas for a flat affine bundle structure on $T M$ which is completely determined by the affine structure of $M$. The resulting flat affine bundle is called $T^{\text {aff }} M$.

Suppose $f: M \rightarrow N$ is an affine map (i.e. $N$ is an affine manifold and $f$ is affine in local charts). Then $T f$, considered as a map $T^{\text {aff }} M \rightarrow T^{\text {aff }} N$, is affine in each fibre, and $T f: T^{\text {aff }} M \rightarrow T^{\text {aff }} N$ is a morphism of flat affine bundles. (Note that in natural affine trivializations, $T_{x} f$ does not appear to be linear.)

Now assume that the affine atlas $\left\{\theta_{i}, U_{i}\right\}$ on $M$ is such that each nonempty map $q_{i j}=\phi_{i} \circ \phi_{j}^{-1}$ extends to a (global) affine automorphism of $\mathbf{E}$ (as when $U_{i} \cap U_{j}$ are connected). Then the collection $\left\{g_{i j}\right\}$ is a cocycle for the flat affine bundle $T^{\text {aff }} M$; and the collection of linear parts $\left\{\operatorname{Lin}\left(g_{i j}\right)\right\}$ is a cocycle for the flat vector bundle TM. 
We now describe the canonical flat vector bundle structures on $T M$. Each fibre $T_{x} M$ coincides in a natural way with the group of translations of $T_{x} M=T^{\text {aff }}{ }_{x} M$. Therefore we identify $T_{x} M$ with the fibre over $x$ of $\left(T^{\text {aff }} M\right)^{L}$, the derived bundle of $T^{\text {aff }} M$ (see $\S 1.3$ ). Thus $T M$ is canonically isomorphic, as a vector bundle, to the flat vector bundle $\left(T^{\text {aff }} M\right)^{L}$.

2.2. Developing sections. Since $T^{\text {aff }} M$ is a flat bundle, it has a canonical foliation $\mathscr{F}$ transverse to its fibres. For $U$ an open set in $\mathbf{E}$, the foliation of $T^{\text {aff }} U=U \times \mathbf{E}$ has as leaves the sets defined by $x+y=$ constant. Notice that these leaves are transverse to the submanifold $U \times\{0\}$.

It follows that the leaves of $\mathscr{F}$ are transverse to the zero section of $T M$. To emphasize its role in the affine structure we refer to this zero section as the developing section of $T^{\text {aff }} M$, denoted by $\sigma_{M}: M \rightarrow T^{\text {aff }} M$. See Goldman [G] for another treatment.

Let $\left(\phi_{i}, U_{i}\right)$ be an affine chart on $M$. In terms of the natural trivialization $\theta_{i}$ of $T^{\text {aff }} U_{i}$ induced by $\phi_{i}$ (see $\left.\$ 2.1\right)$, the developing section $\sigma_{M}$ corresponds to the map

$$
U_{i} \rightarrow U_{i} \times \mathbf{E}, \quad x \mapsto\left(x, \phi_{i}(x)\right) .
$$

Thus the image $\sigma_{M}\left(U_{i}\right)$ corresponds to the graph of $\phi_{i}$.

To an affine manifold $M$ we have associated a flat affine bundle structure $T^{\text {aff }} M$ on $T M$ with its corresponding foliation $\mathscr{F}$, together with a section $\sigma_{M}$ of $T^{\text {aff }} M$ transverse to $\mathscr{F}$. Conversely, let $N$ be a differentiable manifold and suppose $T N$ has a flat affine bundle structure $\alpha$ with corresponding foliation $\mathscr{F}^{\alpha}$, and that $\sigma$ : $N \rightarrow T N$ is a smooth section transverse to $\mathscr{F}^{\alpha}$. Let $U_{i} \subset N$ be a connected open set over which $T^{\alpha} N$ is trivial, so that there is a flat affine bundle map

$$
f_{i}: T^{\text {aff }} U_{i} \approx U_{i} \times \mathbf{E} .
$$

For each leaf $L$ of $\mathscr{F}^{\alpha}, f_{i}$ takes each component of $L \cap T^{\alpha} U_{i}$ into a set of the form $U_{i} \times\{y\}$. From transversality of $\sigma$ to $\mathscr{F}^{\alpha}$ it follows that the composition

$$
\phi_{i}: U_{i} \stackrel{\sigma}{\rightarrow} T^{\alpha} U_{i} \stackrel{f_{i}}{\rightarrow} U_{i} \times \mathbf{E} \rightarrow \mathbf{E}
$$

is an immersion. Give $U_{i}$ the affine manifold structure induced from $\mathbf{E}$ by $\phi_{i}$. Because $T^{\alpha} N$ is a flat affine bundle, $U_{i} \cap U_{j}$ inherits the same affine structure from $U_{i}$ and $U_{j}$. Thus to $T^{\alpha} N$ and $\sigma$ we have associated an affine structure on $N$.

REMARK. An older approach to affine structures on manifolds is through the idea of a linear connection on TM (as in \$1.10) whose curvature and torsion vanish. The developing section is then viewed as an "integral" of the tensor which is the identity endomorphism of TM. This interpretation may be found in Matsushima [Mt], where it is attributed to J. L. Koszul.

2.3. Developing maps. Let $p: \tilde{M} \rightarrow M$ be a universal covering of an affine manifold $M$ modeled on $\mathbf{E}$. We give $M$ the induced affine structure (making $p$ an affine map). Let $\pi$ denote the group of deck transformations of $\tilde{M}$.

A developing map for $M$ is an affine immersion $\tilde{M} \rightarrow \mathbf{E}$.

The following basic result is well known; we include a proof for the reader's convenience. 
THEOREM. (a) There exists a developing map.

(b) For any developing map $f$ there is a unique holonomy homomorphism $h$ : $\pi \rightarrow \operatorname{Aff}(E)$ for which $f$ is equivariant, that is,

$$
f \circ g=h(g) \circ f \quad(g \in \pi) .
$$

(c) If $f^{\prime}$ is another developing map, then $f^{\prime}=\gamma \circ f$ for a unique $\gamma \in \operatorname{Aff}(\mathbf{E})$.

Proof. (a) Let $\psi: T^{\text {aff }} \tilde{M} \rightarrow \mathbf{E}$ be a trivialization. Let $\sigma_{\tilde{M}}: \tilde{M} \rightarrow T^{\text {aff }} M$ be the developing section (= zero section). Then the composition $f=\psi \circ \sigma_{\tilde{M}}: \tilde{M} \rightarrow \mathbf{E}$ is an immersion because the zero section $\sigma_{\tilde{M}}: \tilde{M} \rightarrow T \tilde{M}$ is transverse to leaves of the foliation $\tilde{\mathscr{F}}$ of $T^{\text {aff }} \tilde{M}$ (see $\S 2.2$ ). Since $f$ is easily seen to be affine in local coordinates, $f$ is a developing map.

(b) Let $h: \pi \rightarrow \operatorname{Aff}(E)$ be the holonomy of the flat affine bundle $T^{\text {aff }} M$ (see $\$ 1.2$ ). It is readily proved that $f$ as constructed above is $h$-equivariant. The general case of (b) now follows from (c), and the proof of (c) is trivial. Q.E.D.

We call a homomorphism $h$ as in (b) an affine holonomy homomorphism for $M$. When $\tilde{M}$ is given, $h$ is uniquely determined up to a composition with an inner automorphism of Aff(E).

It is easy to see that an affine holonomy homomorphism for $M$ is also a holonomy homomorphism for the flat affine bundle $T^{\text {aff }} M$ as defined in \$1.2.

We generally pretend the developing map is unique and denote it by dev: $\tilde{M} \rightarrow \mathbf{E}$. By a similar abuse of language the image $h(\pi)$ of the corresponding holonomy homomorphism is called the affine holonomy group $\Gamma$ of $M$. The image $\operatorname{Lin}(\Gamma) \subset$ $\mathrm{GL}(E)$ is called the linear holonomy group of $M$.

An affine manifold $M$ is complete if its developing map is bijective. Equivalently, $\boldsymbol{M}$ is complete if its universal covering is affinely isomorphic to $\mathbf{E}$. For a general discussion of complete affine manifolds we refer to Milnor [Mi] and Fried and Goldman [FG1]; in the latter paper all such structures on compact manifolds of dimension $\leqslant 3$ are classified. If $M$ is a compact complete affine manifold, then its affine holonomy group $\Gamma$ acts freely and properly discontinuously on $\mathbf{E}$ with compact fundamental domain. It is an amusing exercise to show that the converse holds.

2.4. The radiance obstruction of an affine manifold. It will be convenient to denote the flat affine bundle $T^{\text {aff }} M$ by $\tau$ or $\tau_{M}$, and the flat vector bundle $\left(T^{\text {aff }} M\right)^{L}$ by $E$ or $E_{M}$ (see $\left.\$ 2.1\right)$.

As a vector bundle $E_{M}$ is the same as $T M$, but $E_{M}$ has a flat structure. In particular the de Rham cohomology group $H^{*}(M ; E)$ and related groups will be important.

The radiance obstruction of $M$ is defined to be $c_{M}=c_{\tau} \in H^{1}(M ; E)$; thus $c_{M}$ is the de Rham radiance obstruction of $\tau$. Since $E$ and $T M$ are the same as vector bundles, $c_{M}$ is represented by a $T M$-valued 1 -form on $M$, that is, by an endomorphism of $T M$. By $\$ 1.14$ this endomorphism is $\nabla \sigma_{M}$, the covariant differential of the zero section of $T M$. From $\$ \S 2.1$ and $1.13, \nabla \sigma_{M}$ is the identity isomorphism of $T M$. 
This proves

THEOREM. The radiance obstruction of $M$ is the de Rham class $c_{M} \in H^{1}(M ; E)$ represented by the identity endomorphism of $T M$, where $E$ denotes the flat vector bundle structure on TM.

2.5. Parallel and polynomial tensors. A tensor (field) on a connected affine manifold $M$ is called parallel if in affine coordinates each component of the tensor is constant. Alternatively, a parallel tensor field on $M$ is a flat section of the bundle of tensors on $M$, which is given the flat vector bundle structure induced from $\left(T^{\text {aff }} M\right)^{L}$.

Let $\mathbf{t}$ be a parallel tensor on $M$ and $\mathfrak{t}$ the induced form on the universal cover $\tilde{M}$. Fix a developing map dev: $\tilde{M} \rightarrow E$. Let the corresponding holonomy homomorphism be $h: \pi \rightarrow \operatorname{Aff}(\mathbf{E})$. Let $\Gamma \subset \operatorname{Aff}(\mathbf{E})$ and $\Lambda \subset \mathrm{GL}(\mathbf{E})$ denote the affine and linear holonomy groups.

It is easy to prove (see e.g. [GH2]) that there is a unique (constant) tensor $\mathbf{t}_{\mathbf{E}}$ on $\mathbf{E}$ which is related to $t$ by dev; and $t_{E}$ is invariant under the induced action of $\Lambda$ on tensors. Conversely, for any $\Lambda$-invariant tensor $\mathbf{t}^{\prime}$ on $\mathbf{E}$ there is a unique parallel tensor $\mathbf{t}$ on $M$ such that $\mathbf{t}^{\prime}=\mathbf{t}_{\mathrm{E}}$ (defined as above).

More generally any tensor $\mathbf{s}$ on $M$ corresponds to a unique $\Gamma$-invariant tensor $\mathbf{s}^{\prime}$ on $\operatorname{dev}(\tilde{M})$ which is related by $\operatorname{dev}$ to the lift $\tilde{\mathbf{s}}$ of $\mathbf{s}$ to $\tilde{M}$. In particular if the components of $\mathbf{s}$ are given by polynomial maps $\mathbf{E} \rightarrow \mathbf{R}$ in local affine coordinates, then $\mathbf{s}^{\prime}$ extends to a $\Gamma$-invariant polynomial tensor field on $\mathbf{E}$.

2.6. Parallel differential forms and the evaluation formula. From $\$ 2.5$ it follows that a parallel exterior $k$-form $\omega$ on $M$ corresponds to a linear map $\Lambda^{k} \mathbf{E} \rightarrow \mathbf{R}$ which is invariant under the action of the linear holonomy group of $M$ on $\Lambda^{k} \mathbf{E}^{*}$. Thus the vector space of parallel $k$-forms on $M$ is $H\left(M ; \Lambda^{k} E^{*}\right)$, where $E$ is the flat tangent vector bundle of $M$ (see $\S \S 2.1,2.4), \Lambda^{k} E^{*}$ is the (fibrewise) $k$ th exterior power of its dual bundle (also a flat vector bundle over $M$ ), and $H^{0}$ denotes the vector space of flat sections (equivalently, the zeroth de Rham cohomology).

There is a natural fibrewise pairing of bundles $\Lambda^{k} E^{*} \oplus \Lambda^{k} E \rightarrow M \times \mathbf{R}$ which over $x \in M$ is simply the duality pairing. Considering this as a coefficient pairing to the trivial flat line bundle, we obtain a natural pairing

$$
K: H^{0}\left(M ; \Lambda^{k} E^{*}\right) \times H^{k}\left(M ; \Lambda^{k} E\right) \rightarrow H^{k}(M ; \mathbf{R}),
$$

also denoted $K(\alpha, \beta)=\langle\alpha, \beta\rangle$.

The following useful formula combining parallel forms, the radiance obstruction, and the real cohomology of $M$, will be applied many times. We call it the evaluation formula: it says the cohomology class of a parallel differential $k$-form $\omega$ is obtained by evaluating (via $K$ ) $\omega$ on the $k$ th exterior power of the radiance obstruction:

Proposition. Let $\omega \in H^{0}\left(M ; \Lambda^{k} E^{*}\right)$ be a parallel $k$-form. Then $\left\langle\omega, \Lambda^{k} c_{M}\right\rangle=[\omega]$, the de Rham cohomology class of $\omega$.

Proof. By Theorem $2.4, \Lambda^{k} c_{M}$ is represented by the identity endomorphism of $\Lambda^{k}(T M)$. The lemma follows from this by working through the definition of $K$. Q.E.D.

The following corollary is a basic principle which will be used throughout the rest of this work. 
THEOREM. Let $\omega$ be a parallel $k$-form on the affine manifold $M$ with nonzero cohomology class $[\omega] \in H^{k}(M ; \mathbf{R})$. Then the kth exterior power of the radiance obstruction

$$
\Lambda^{k} c_{M} \in H^{k}\left(M ; \Lambda^{k} \mathbf{E}\right)
$$

is nonzero. Q.E.D.

Theorem 3.2 of [FGH2] treats the special case of $k=1$ in this theorem. The same paper exhibits a compact affine 3-manifold having a nonzero parallel 2-form, but whose radiance obstruction vanishes (the case $k=1 / 2$ on p. 511 of [FGH2]). By the theorem above the cohomology class of such a form must vanish; compare [GH2 and GHL].

In the next three sections we give some applications of the evaluation formula.

2.7. Parallel volume. The $n$-dimensional affine manifold $M$ is said to have a parallel volume form $\omega$ if $M$ is orientable and $\omega$ is a parallel nonzero exterior form of degree $n$. In any affine chart, $\omega$ appears as a nonzero constant multiple of the Euclidean volume form $d x_{1} \wedge \cdots \wedge d x_{n}$. The existence of $\omega$ is equivalent to the linear holonomy group lying in $\mathrm{SL}(\mathbf{E})$.

If $M$ is nonorientable, then we say $M$ has parallel volume if its oriented double covering has a parallel volume form.

The following old and unsolved conjecture of $L$. Markus motivates many of the results of this paper.

L. MARKus' Conjecture. A compact affine manifold is complete if and only if it has parallel volume.

In [FGH2] Markus' conjecture was proved for the case of nilpotent holonomy, generalizing an earlier result of Smillie [Sm2] for abelian holonomy. Further cases of the conjecture are proved below.

Applying the evaluation formula (see \$2.6) to a parallel volume form proves the following important fact.

THEOREM. If $M$ is an orientable n-dimensional compact affine manifold with parallel volume, then $\Lambda^{n} c_{M} \neq 0$. Q.E.D.

This sharpens Theorem 3.1 of [FGH2] which concludes only that $c_{M} \neq 0$.

The following well known result is useful.

Proposition. Suppose the linear holonomy of $M$ factors through a group $G$ which admits no nontrivial homomorphism to the group $\mathbf{R}$ of real numbers. Then $M$ has parallel volume. In particular if the first Betti number of $M$ is zero then $M$ has parallel volume.

Proof. Let the linear holonomy $\pi \rightarrow \operatorname{GL}(\mathbf{E})$ factor through $G$ and consider the composition

$$
\pi \stackrel{f}{\rightarrow} G \stackrel{g}{\rightarrow} \mathrm{GL}(\mathbf{E}) \stackrel{j}{\rightarrow} \mathbf{R}
$$

where $j(\gamma)=\log \mid$ Det $\gamma \mid$ and $g f$ is the linear holonomy. Since $j g=0$ it follows that every element of the linear holonomy group has determinant \pm 1 . Q.E.D. 
REMARK. Markus' conjecture implies nontrivial information on the topology of affine manifolds. Suppose $M$ is a compact manifold which admits an affine structure. Then Markus' conjecture has the surprising consequence that either the first Betti number of $M$ is nonzero or $M$ is an aspherical space (and indeed covered by Euclidean space).

2.8. Irreducible affine holonomy. An affine representation $h: \pi \rightarrow \operatorname{Aff}(\mathbf{E})$ is irreducible if no proper affine subspace is invariant under $h(\pi)$.

LEMMA. Let $M$ be an affine manifold having a parallel $k$-form which represents a nonzero cohomology class in $H^{k}(M ; \mathbf{R})$. Then the affine holonomy group $\Gamma$ of $M$ cannot preserve any affine subspace of dimension $m<k$.

Proof. If $\Gamma$ preserves an $m$-dimensional affine subspace, then $T^{\text {aff }} M$ has a flat affine subbundle of fibre dimension $m$, and by $\S 1.15$ we then have $\Lambda^{j} c_{M}=0$ for all $j>m$. Since $\Lambda^{k} c_{M} \neq 0$ by Theorem 2.7, it follows that $k \leqslant m$. Q.E.D.

THEOREM. The affine holonomy of a compact affine manifold $M$ with parallel volume is irreducible.

Proof. Passing to an oriented covering we assume $M$ has a parallel volume form. Then $\Lambda^{n} c_{M} \neq 0$ by $\$ 2.7$ and the theorem follows from the lemma above. Q.E.D.

This theorem was proved in [FGH2] under the extra assumption that $\Gamma$ is nilpotent. In [GH3] we generalize it by showing that no proper semialgebraic set is invariant under $\Gamma$.

In [FGH2, p. 496], it is shown that the affine holonomy is irreducible for any compact complete affine manifold.

2.9. Radiant manifolds. The affine manifold $M$ is called radiant when $c_{M}=0$.

Suppose $M$ is radiant. Then $T^{\text {aff }} M$ has a global flat section. Therefore by $\S 1.5$ any affine holonomy group $\Gamma$ has at least one stationary point in $\mathbf{E}$.

Let $p: \tilde{M} \rightarrow M$ be a universal covering. We can always choose a developing map $\operatorname{dev}: \tilde{M} \rightarrow E$ so that the corresponding holonomy group has the origin in $\mathbf{E}$ as a stationary point. In this case the affine and linear holonomy representations coincide.

Suppose $\Gamma$ fixes the origin. Let $\left\{U_{i}\right\}$ be an open covering of $M$ such that there are open sets $V_{i} \subset \tilde{M}$ which map diffeomorphically onto $U_{i}$ by $p$, and which map diffeomorphically by dev. For each $i$ define an affine chart

$$
\phi_{i}=\operatorname{dev} \circ\left(p \mid V_{i}\right)^{-1}: U_{i} \rightarrow \mathbf{E} .
$$

These charts form an affine atlas for $M$ whose coordinate changes are linear. The existence of such an atlas is equivalent to the radiance of $M$.

There are radiant manifolds diffeomorphic to $S^{1} \times V$, where $V=S^{n-1}$ (the Hopf manifolds), or $V$ is any compact surface (see [FGH2, p. 502]).

THEOREM. Let $M$ be a compact radiant manifold. Then:

(a) $M$ does not have parallel volume;

(b) the first Betti number of $M$ is nonzero;

(c) every parallel 1-form on $M$ is zero; 
(d) the Euler characteristic of $M$ is zero;

(e) the developing map of $M$ is not surjective.

Parts (a), (c), (d), and (e) are proved in [FGH2, §3]. The proofs all involve the radial vector field on $\mathbf{E}$ which is $\Gamma$-invariant and vanishes only at the origin. In [FGH2] compactness of $M$ is used to show that the induced vector field $R_{M}$ on $M$ is nonsingular. This proves (d) and (e). Further use of the vector field $R_{M}$ proves (a) and (c). Part (b) follows from (a) and Proposition 2.7. Q.E.D.

2.10. Parallel forms and nilpotent holonomy. Let $M$ be a compact affine manifold. In this section we assume the affine holonomy group $\Gamma$ of $M$ is nilpotent. For background on this class of affine manifolds, see [FGH2].

THEOREM. Suppose $M$ has a parallel $k$-form $\omega$ whose cohomology class $[\omega] \in$ $H^{k}(M ; \mathbf{R})$ is nonzero. Then $M$ has a nonzero parallel $j$-form for all $j \leqslant k$.

Proof. By the evaluation formula (\$2.6) we know $\Lambda^{k} c_{M} \neq 0$, so $\Lambda^{j} c_{M} \neq 0$ for all $j \leqslant k$. For these $j$, therefore, $H^{j}\left(\Gamma ; \Lambda^{j} \mathbf{E}\right) \neq 0$, where $\mathbf{E}$ is considered as a $\Gamma$-module via the linear representation $\Gamma \subset \operatorname{Aff}(\mathbf{E}) \rightarrow \mathrm{GL}(\mathbf{E})$.

For any $\Gamma$-module $\mathrm{V}$ it is known that, because of nilpotency of $\Gamma, H^{j}(\Gamma ; \mathrm{V}) \neq 0$ implies $H^{0}\left(\Gamma ; \mathbf{V}^{*}\right) \neq 0$ (see [FGH2, §1]; also Hirsch [H] and Dwyer [Dw]). Taking $V=\Lambda^{j} \mathbf{E}$ completes the proof. Q.E.D.

In [GHL] it is proved that when $M$ is nonradiant there always exists a cohomologically nontrivial parallel $k$-form, where $k>0$ is the Fitting dimension of $M$ : the dimension of the largest invariant linear subspace of $\mathbf{E}$ on which the linear holonomy is unipotent. In [GH3] we study the Fitting dimension in terms of orbits of algebraic groups.

The parallel $j$-form in the theorem cannot always be taken to be nonzero in cohomology. For example consider the complete, compact affine 3-manifold $M=$ $\mathbf{R}^{3} / \Gamma$, where $\Gamma$ is the nilpotent subgroup of $\operatorname{Aff}\left(\mathbf{R}^{3}\right)$ of all affine transformations of the form

$$
\left[\begin{array}{ccc}
1 & 6 c & 18 c^{2} \\
0 & 1 & 6 c \\
0 & 0 & 1
\end{array}\right]\left[\begin{array}{l}
a \\
b \\
c
\end{array}\right]
$$

where $a, b, c$ are integers. Every $\Gamma$-invariant parallel 2 -form on $\mathbf{R}^{3}$ is a constant multiple of $d y \wedge d z$, where $(x, y, z)$ are the linear coordinates in $\mathbf{R}^{3}$. Now $6 d y \wedge d z$ $=d(\omega)$, where $\omega=d x-6 z d y+18 z^{2} d z$ is $\Gamma$-invariant. Therefore every parallel 2 -form on $M$ is exact.

2.11. Cohomological dimension. In this section and the next we prove some results due in essence to J. Smillie. The top-dimensional cohomology class, which we call $\Lambda^{n} c_{M}$, was discovered independently by him; see [Sm2]. At this writing some of Smillie's results have not been published.

The real cohomological dimension $\operatorname{rcd}(G)$ of a group $G$ is the smallest integer $k$ such that $H^{i}(G ; \mathbf{F})=0$ for all $i>k$ whenever $\mathbf{R}$ is a finite-dimensional real vector space with a $G$-module structure. 
It can be shown that $[G: H]<\infty$ implies $\operatorname{rcd}(G) \leqslant \operatorname{rcd}(H)$, and that $\operatorname{rcd}\left(F^{*} G\right) \leqslant$ $\max \{\operatorname{rcd}(F), \operatorname{rcd}(G)\}$, where ${ }^{*}$ denotes free product. If there is an EilenbergMac Lane complex $K(G, 1)$ of dimension $\leqslant k$ then $\operatorname{rcd}(G) \leqslant k$.

It is easy to see that $\operatorname{rcd}(G)$ is less than or equal to the virtual cohomological dimension of $G$ (see Serre [Se]).

Smillie [Sm2] shows that $\operatorname{rcd}(G) \leqslant 1$ if $G$ is built up from finite and infinite cyclic groups by a finite number of free products and finite extensions. He used this fact to exclude certain manifolds from having affine structures with parallel volume, e.g. a connected sum of manifolds homeomorphic to $S^{1} \times S^{2}$.

The following result generalizes Smillie's theorem.

THEOREM. If an affine manifold $M$ has a nonzero cohomology class represented by a parallel exterior $k$-form $\omega$, then its affine holonomy $h$ cannot factor through a group $G$ having $\operatorname{rcd}(G)<k$.

Proof. The existence of $\omega$ implies by $\S 2.6$ that $\Lambda^{k} c_{h} \neq 0$. Suppose $h$ is a composite homomorphism

$$
h: \pi \stackrel{f}{\rightarrow} G \stackrel{g}{\rightarrow} \operatorname{Aff}(\mathbf{E}) .
$$

Then $f^{*}\left(\Lambda^{k} c_{g}\right)=\Lambda^{k} c_{h} \neq 0$, whence $\Lambda^{k} c_{g}$ is a nonzero cohomology class of $G$ with coefficients in the $G$-module defined by the linear part of $g$. Therefore $\operatorname{rcd}(G) \geqslant k$. Q.E.D.

Taking $\omega$ in the theorem above to be a parallel volume form proves

COROLlARY. Let $M$ be a compact affine manifold having parallel volume. Then the affine holonomy of $M$ cannot factor through a group $G$ having $\operatorname{rcd}(G)<\operatorname{dim} M$.

This result can be used to rule out various groups from being the fundamental or holonomy groups of certain kinds of affine manifolds. For example, the fundamental group of a surface has $\mathrm{rcd} \leqslant 2$. Therefore, it cannot be the fundamental or holonomy group of a compact manifold $M$ with parallel volume if $\operatorname{dim} M>2$ (as is seen by taking $\omega$ to be a volume form).

2.12. Radiance dimension and parallel cohomology. Let $H_{\mathrm{par}}^{k}(M ; \mathbf{R})$ denote the subspace of $H^{k}(M ; \mathbf{R})$ of de Rham classes which contain parallel $k$-forms. Let $\mathscr{U}$ be a radiant cover of $M$, i.e. $\mathscr{U}$ is a cover by open sets, each of which is radiant in its induced affine structure. Let $N \mathscr{U}$ denote the simplicial complex which is the nerve of $\mathscr{Q}$.

THEOREM. The inclusion $H_{\mathrm{par}}^{k}(M ; \mathbf{R}) \rightarrow H^{k}(M ; \mathbf{R})$ factors through the natural map $H^{k}(N \mathscr{U} ; \mathbf{R}) \rightarrow H^{k}(M ; \mathbf{R})$.

Proof. By $\S 1.9$ the radiance obstruction $c_{M} \in H^{1}(M ; E)$ comes from a Čech class $c_{\mathscr{Q}} \in H^{1}(\mathscr{Q} ; E)$.

Let a class $[\omega] \in H_{\mathrm{par}}^{k}(M ; \mathbf{R})$ be represented by the parallel $k$-form $\omega$. We consider $\omega$ as lying in $H^{0}\left(M ; \Lambda^{k} E^{*}\right)$. Since each element of $\mathscr{U}$ is radiant, $\omega$ also corresponds to a Čech class $\omega_{\mathscr{q}} \in H^{0}\left(\mathscr{Q} ; \Lambda^{k} E^{*}\right)$. There is a natural pairing

$$
H^{k}\left(\mathscr{U} ; \Lambda^{k} E\right) \otimes H^{c}\left(\mathscr{U} ; \Lambda^{k} E^{*}\right) \rightarrow H^{k}(\mathscr{U} ; \mathbf{R})
$$


corresponding to the coefficient pairing $\Lambda^{k} E \otimes \Lambda^{k} E^{*} \rightarrow \mathbf{R}$. This pairing takes $\Lambda^{k}\left(c_{M}\right) \otimes \Omega_{\mathscr{Q}}$ to an element $j[\omega] \in H^{k}(\mathscr{U} ; \mathbf{R})=H^{k}(N \mathscr{U} ; \mathbf{R})$ which depends only on

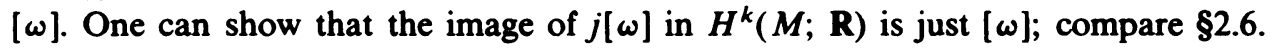
Q.E.D.

Define the radiance dimension $\operatorname{rad}(M)$ to be the smallest integer $d$ such that there exists a radiant cover $\mathscr{Q}$ of $M$ with $H^{i}(N \mathscr{U} ; \mathbf{R})=0$ for $i>d$.

COROLLARY. If $M$ admits a parallel $k$-form which is nonzero in cohomology then $\operatorname{rad}(M) \geqslant k$. Q.E.D.

It seems likely that the resulting inclusion $H_{\mathrm{par}}^{*}(M) \rightarrow H^{*}(N \mathscr{U})$ constructed above is a homomorphism of graded algebras; but we have not proved this.

2.13. Fibrations with radiant fibres. Throughout this section $f: M \rightarrow B$ denotes a locally trivial fibration of an affine manifold $M$ over a topological manifold $B$. We assume that the affine holonomy homomorphism of $M$ vanishes on the image of the fundamental group of the fibre.

The definition of $\operatorname{rad}(M)$ is given in $\$ 2.12$.

Proposition. (a) The affine holonomy of $M$ factors through $\pi_{1}(B)$;

(b) $\operatorname{rad}(M) \leqslant \operatorname{dim} B$.

Proof. (a) follows from part of the exact homotopy sequence of the fibration $f$ :

$$
\pi_{1}\left(f^{-1}(x)\right) \rightarrow \pi_{i}(M) \rightarrow \pi_{i}(B) \rightarrow 0 .
$$

(b) follows by considering covers of $M$ having the form $\left\{f^{-1} V_{i}\right\}$, where $\left\{V_{i}\right\}$ is a covering of $B$ by simply connected open sets over each of which the fibration is trivial, and whose nerve has the same dimension as $B$. Q.E.D.

COROLLARY. Let $\omega$ be a parallel $k$-form on M.Then $\omega$ is zero in cohomology if either

(a) $k>\operatorname{rcd}\left(\pi_{1}(B)\right)$, or

(b) $k>\operatorname{dim} B$.

Proof. (a) follows from (a) of the proposition and \$2.11, while (b) follows from (b) of the proposition and \$2.12. Q.E.D.

As an application we obtain

THEOREM. Let $B$ and $N$ be compact manifolds, with $\pi_{1}(N)$ finite. Then:

(a) $B \times N$ cannot have an affine structure with parallel volume.

(b) Assume also that $H^{1}(B ; \mathbf{R})=0$. Then $B \times N$ cannot have any affine structure.

Proof. $\pi_{1}(N)$ being finite means the product fibration $B \times N \rightarrow B$ fulfills the hypothesis of this section. Therefore (a) follows from (b) of the proposition and §2.12. And (b) now follows because if $H^{1}(B \times N ; \mathbf{R})=0$ then any affine structure on $B \times N$ would necessarily have parallel volume, contradicting (a). Q.E.D.

In a forthcoming paper [FG2], Proposition 2.13 is used to show that a 3-dimensional Seifert manifold admits a flat Lorentz metric if and only if it is covered by a $T^{2}$-bundle over $S^{1}$. See also [FG1]. 
2.14. Affinely rigid groups. We call a discrete group $\Pi$ affinely rigid if $H^{1}(\Pi ; E)=0$ for all real finite-dimensional $\Pi$-modules $E$; a simple example is provided by any finite group.

In [Mg] Margulis proved a remarkable theorem which implies that $\Pi$ is affinely rigid if it is an irreducible lattice in a semisimple Lie group $G$ of $\mathbf{R}$-rank $\geqslant 2$. We call such groups $\Pi$ Margulis groups. An example is $\operatorname{SL}(Z, n)$ for any $n \geqslant 3$.

Margulis proved that any linear representation of such a $\Pi$ either takes values in a compact subgroup, or else extends to a representation of $G$; and the same holds for affine representations (see \$1.7). (For another proof of Margulis' theorem see Zimmer [Z].) Now every representation of a compact group, or of a semisimple group (see Milnor [Mi]), is radiant; and this property is equivalent to affine rigidity.

By taking $E=\mathbf{R}$ we see that any affine representation of an affinely rigid group must be volume-preserving.

THEOREM. The affine holonomy of a compact affine manifold cannot factor through an affinely rigid group. In particular it cannot factor through a Margulis group.

Proof. If the holonomy did so factor, the manifold would have to be radiant, and would have to have parallel volume by Proposition 2.7. But it is impossible for a compact radiant manifold to have parallel volume (see [FGH2, 3.1]). Q.E.D.

By an obvious extension of a theorem of J. Smillie about free products of finite groups [Sm2] one obtains a nonfactoring theorem for free products of affinely rigid groups:

Proposition. Let $M$ be a compact affine manifold. If $\operatorname{dim} M>m$ then the affine holonomy of $M$ cannot factor through the free product of $m$ affinely rigid groups.

Proof. Suppose the holonomy factors through $\Pi=\Pi_{1} * \cdots * \Pi_{m}$, each $\Pi_{i}$ being affinely rigid. The classifying space $B \Pi$ for $\Pi$ can be taken as the one-point union (wedge) of those for the $\Pi_{i}$. A well-known construction using a classifying map $f$ : $M \rightarrow B \Pi_{1} \vee \cdots \vee B \Pi_{m}$ for $T^{\text {aff }} M$ produces an open cover $\left\{U_{1}, \ldots, U_{m}\right\}=\mathscr{U}$ of $M$ such that $f\left(U_{i}\right) \subset B \Pi_{i}$. This implies that the affine holonomy of $U_{i}$ factors through $\Pi_{i}$. Therefore $\mathscr{Q}$ is a radiant cover. Thus $\operatorname{rad}(M)<\operatorname{dim} M$ so $M$ cannot have parallel volume by $\$ 2.12$. But any homomorphism $\Pi \rightarrow \mathbf{R}$ must vanish on each $\Pi_{i}$, so $M$ must have parallel volume by Proposition 2.7. This contradiction completes the proof. Q.E.D.

ACKNowledgement. It is a pleasure to thank D. Fried, S. Kobayashi and J. Smillie for many helpful and stimulating conversations. We are especially grateful to John Smillie for sharing his results with us, some of which appear here for the first time.

\section{REFERENCES}

[AM] L. Auslander and L. Markus, Holonomy of flat affinely connected manifolds, Ann. of Math. (2) 62 (1955), 139-159.

[Br] G. Bredon, Sheaf theory, McGraw-Hill, New York, 1967.

[Dw] W. G. Dwyer, Vanishing cohomology over nilpotent groups, Proc. Amer. Math. Soc. 49 (1975), $8-12$. 
[FG1] D. Fried and W. Goldman, Three-dimensional affine crystallographic groups, Adv. in Math. 47 (1983), 1-49.

[FG2] (in preparation).

[FGH1] D. Fried, W. Goldman and M. Hirsch, Affine manifolds and solvable groups. Bull. Amer. Math. Soc. 3 (1980), 1045-1047.

[FGH2] __ Affine manifolds with nilpotent holonomy, Comment. Math. Helv. 56 (1981), 487-523.

[G] W. Goldman, Discontinuous groups and the Euler class, Doctoral Dissertation, Univ. of California, Berkeley, Calif., 1980.

[GH1] W. Goldman and M. Hirsch, A generalization of Bieberbach's theorem, Invent. Math. 65 (1981), $1-11$.

[GH2] __ Polynomial forms on affine manifolds, Pacific J. Math. 101 (1982), 115-121.

[GH3] __ Affine structures and actions of Lie groups (in preparation).

[GHL] W. Goldman, M. Hirsch and G. Levitt, Invariant measures for affine foliations, Proc. Amer. Math. Soc. 86 (1982), 511-518.

[H] M. W. Hirsch, Flat manifolds and the cohomology of groups, Algebra and Geometric Topology, Lecture Notes in Math., vol. 664, Springer-Verlag, Berlin and New York, 1977.

[HT] M. W. Hirsch and W. Thurston, Foliated bundles, flat manifolds and invariant measures, Ann. of Math. (2) 101 (1975), 369-390.

[KN] S. Kobayashi and K. Nomizu, Foundations of differential geometry. Vol. I, Interscience, New York, 1963.

[Mg] G. A. Margulis, Discrete groups of motions of spaces of nonpositive curvature, Trans. Amer. Math. Soc. 109 (1977), 33-45.

[Mi] J. W. Milnor, On fundamental groups of complete affinely flat manifolds, Adv. in Math. 25 (1977), 178-187.

[Mt] Y. Matsushima, Affine structures on complex manifolds, Osaka J. Math. 5 (1968), 215-222.

[Mk] L. Markus, Cosmological models in differential geometry, Mimeographed Notes, Univ. of Minnesota, 1962, p. 58.

[Se] J. P. Serre, Cohomologie des groupes discrets, Prospects in Mathematics, Ann. of Math. Studies, no. 70, Princeton Univ. Press, Princeton, N. J., 1971, pp. 77-169.

[Sm1] J. Smillie, Affinely flat manifolds, Doctoral Dissertation, Univ. of Chicago, 1977.

[Sm2] _ An obstruction to the existence of affinely flat manifolds, Invent. Math. 64 (1981), 411-415.

[Z] R. J. Zimmer, Ergodic theory, group representations, and rigidity, Bull. Amer. Math. Soc. (N.S.) 6 (1982), 383-416.

Department of Mathematics, Massachusetts institute of Technology, Cambridge, MasSACHUSETTS 01239

Department of Mathematics, University of California, Berkeley, California 94720 